\title{
Multiple Massive Bladder Calculi Following Traumatic Pelvic Fracture
}

\author{
Ozgu Aydogdu ${ }^{1, *}$, Caner Alptekin ${ }^{2}$ \\ ${ }^{1}$ Department of Urology, Medical Park Hospital, Izmir, Turkey \\ ${ }^{2}$ Department of Urology, Bornova State Hospital, Izmir, Turkey \\ *Corresponding author: ozgucan@yahoo.com
}

Received December 13, 2012; Revised January 17, 2013; Accepted February 16, 2013

\begin{abstract}
A 25 year old man was hospitalized with the complaints of pain during micturition and macroscopic hematuria. Plain radiography showed 4 properly shaped bladder stone shadows. Cystolithotomy was performed following cystoscopy and bladder neck incision. The stones weighed $500 \mathrm{~g}$ totally. Previously we have reported a similar patient with giant bladder calculi [1]. To the best of our knowledge the present patient represents one of the largest bladder stone cases reported to date.
\end{abstract}

Keywords: bladder, calculi, pelvic trauma

\section{Case report}

We present a 25 year old man visited our outpatient clinic with the chief complaints of dysuria and macroscopic hematuria. Our patient had a history of traffic accident injury resulted as internal fixation for pelvic fracture 2 years ago. He has had lower urinary tract symptoms including urinary frequency, urgency, voiding difficulty, and urinary intermittency similar to our patient reported previously [1]. His physical examination was normal and plain radiography revealed internal fixators and 4 properly shaped pelvic calculi (Figure 1).

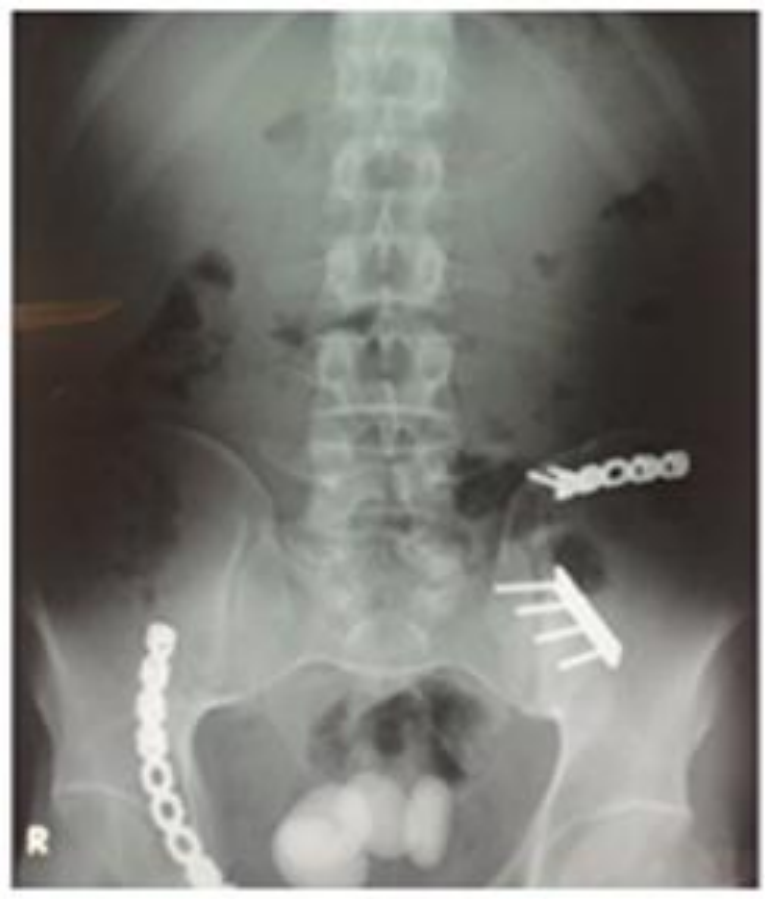

Figure 1. Four properly shaped massive pelvic calculi seen indirect graphy
Blood urea nitrogen and serum creatinin levels were 17 and $0.8 \mathrm{mg} / \mathrm{dl}$ respectively. Preoperative neurological examination was normal and there were no signs of neurogenic bladder. No additional upper urinary tract calculi were detected on plain radiography and urinary ultrasound scan. We performed cystoscopic examination previous to open surgery on the same operative day. Bladder neck was fibrotic and high in cystoscopy and bladder neck incision with cold knife was performed endoscopically before cystolithotomy operation. All stones were extracted massively. The stones weighed $500 \mathrm{~g}$ totally (Figure 2). All stones had 4 compartments of stratified lamellae composed of calcium phosphate and magnesium ammonium phosphate.

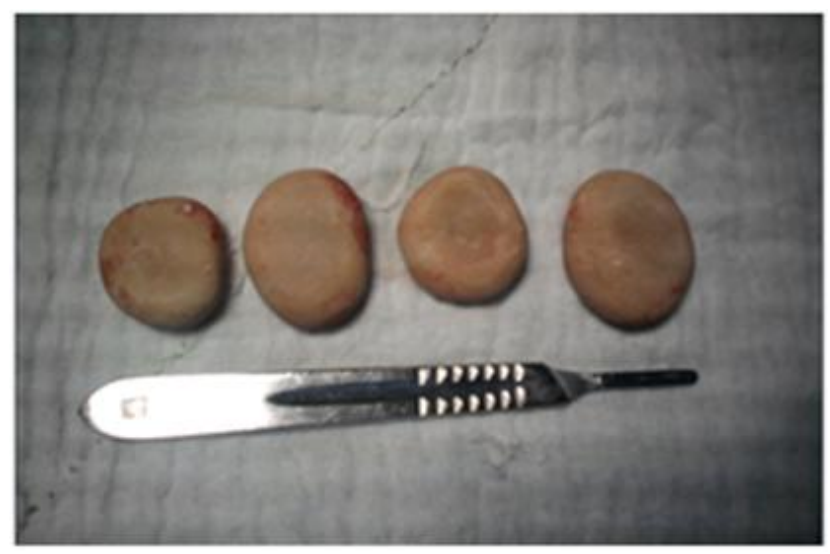

Figure 2. Bladder stones weighed totally $500 \mathrm{~g}$ were extracted by open cystolithotomy

The postoperative period was uneventful. Urethral catheter was removed on the postoperative $6^{\text {th }}$ day and the patients urinary output was normal. The patient was discharged on the postoperative $6^{\text {th }}$ day. He was evaluated in our outpatient clinic at the postoperative $20^{\text {th }}$ day and no residual stone and urinary system dilatation was detected on plain radiography and ultrasound scan. Uroflowmetry was normal. 


\section{Discussion}

Bladder calculi account for 5\% of urinary calculi commonly occurring because of bladder outlet obstruction, neurogenic voiding dysfunction, urinary tract infection or foreign bodies [1,2,3]. Males are more affected than females [1].

Massive bladder calculi are not very frequent in the recent urological practice. Bladder calculi are usually observed secondary to bladder outlet obstruction causing recurrent urinary tract infections, urinary retention, urinary urgency, frequency and macroscopic hematuria. Massive or large bladder stones without upper urinary tract calculi can potentially be observed following pelvic trauma as in our patient. To the best of our knowledge, our patient represents one of the most massive bladder stone cases reported to date.

\section{Conclusion}

Massive or giant bladder calculi can potentially be observed in patients with lower urinary tract symptoms and a history of previous pelvic trauma. Simple diagnostic methods including plain radiography and ultrasound scan can easily demonstrate possible large bladder stones. Surgical intervention with cystolithotomy has satisfactory results for patients presented with massive bladder stones without upper urinary tract calculi.

\section{References}

[1] Aydogdu O, Telli O, Burgu B, Beduk Y. Infravesical obstruction results as giant bladder calculi. Can Urol Assoc J. 2011 Aug;5(4):E77-8. doi: 10.5489/cuaj.10130.

[2] Hammad FT, Kaya M, Kazım E. Bladder calculi: did the clinical Picture change? Urology 2006; 67: 1154-8.

[3] Rahman M, Uddin A, Das GC, et al. A giant vesical calculus. Mymensingh Med J 2007; 16 (2 Suppl): 57-9. 